\section{Where ends the TRAIL in arthritis?}

\author{
Najoua Lalaoui,,,2 Rachel Audo, ${ }^{4}$ \\ Jacques Morel, ${ }^{3,4}$ Olivier Micheau, ${ }^{1,2}$ \\ Michael Hahne ${ }^{4}$ \\ 'INSERM, U866, Dijon, France; \\ 'Université de Bourgogne, Dijon, France; \\ ${ }^{3}$ Service d'Immuno-Rhumatologie et \\ Université Montpellier 1, Montpellier, \\ France; ${ }^{4}$ Institut de Génétique \\ Moléculaire de Montpellier, CNRS \\ UMR5535, Montpellier, France
}

\section{Abstract}

A hallmark of rheumatoid arthritis (RA) is the pseudo-tumoral expansion of fibroblastlike synoviocytes (FLS), as these cells invade and finally destroy the joint structure. RA FLS have been proposed therefore as a therapeutic target. The TNF-related apoptosis-inducing ligand (TRAIL) has gained much attention as a possible therapeutic reagent for the treatment of tumors, as TRAIL was described originally to induce apoptosis specifically in cancer cells but not in normal cells. The fact that FLS in RA patients exhibit tumor-like features led to investigations on the effect of TRAIL on $e x$ vivo RA FLS. In this review we aim to summarize what is presently known on the role of TRAIL in RA.

\section{Introduction}

Rheumatoid arthritis (RA) is a systemic inflammatory disease affecting the joint lining tissue called synovium. The synovium is normally a relatively acellular structure with a delicate intimal lining that is one or two cell layers thick. The rheumatoid synovial tissue is characterized by hyperproliferation of fibroblast-like synoviocytes (FLS) in the intimal lining layer and infiltration of the sublining by macrophages, and T- and B-cells, which promote inflammation and destruction of bone and cartilage. The hyperplasia of synovial fibroblasts in RA contributes to joint destruction, directly through enhanced production of matrix-degrading enzymes, and indirectly through excessive release of proinflammatory cytokines. The proliferation of RA FLS is considered to be pseudo-tumoral, as RA FLS proliferate in an anchorage-independent manner, lack contact inhibition, and express oncogenes and cell cycle proteins indicative of transformation. ${ }^{1}$ Besides an increased proliferation, insufficient apoptosis might contribute to the increased numbers of synovial fibroblasts in RA joints, as apoptosis plays a crucial role in controlling cell numbers by eliminating old cells, unnecessary cells, and unhealthy cells. ${ }^{2}$ Therefore one strategy to treat rheumatoid arthritis is the design of drugs that can restore the normal apoptotic pathways in synovial fibroblasts. $^{2}$

Ligands of the tumor necrosis factor (TNF) family are trimeric cytokines that have an important role in inducing various biological responses such as cell proliferation, differentiation, survival, and apoptosis. ${ }^{3}$ TNF-like ligands capable of inducing apoptosis by binding to their cognate receptors, so-called death receptors, include TNF itself, Fas (CD95) ligand, and the TNF-related apoptosis-inducing ligand (TRAIL). The death receptors of the TNF family are potential targets for inducing apoptosis in malignant cells. In recent years, considerable attention has been focused on the potential benefits of TRAIL in cancer therapy, as a broad range of cancer cells are sensitive to TRAIL-induced apoptosis. ${ }^{4}$ In addition, the use of TRAIL in combination with chemotherapeutic agents or irradiation strengthens its apoptotic effects and frequently sensitizes otherwise TRAIL-resistant tumor cells. Importantly, TRAIL-exposure shows no toxic side effects of therapeutically relevant doses in primates ${ }^{5}$ or in humans. ${ }^{6}$ Likewise agonistic antibodies targeting either DR4 or DR5 are well tolerated in patients. ${ }^{7-11}$ The fact that FLS in RA patients exhibit tumor-like features led to investigations on the effect of TRAIL in RA. In this review we aim to summarize what is presently known about the role of TRAIL in RA.

\section{TRAIL in general}

TRAIL is a type II membrane ligand that belongs to the TNF super-family, and is mainly known to its ability to trigger cell death in a somewhat tumor selective manner. ${ }^{12}$ Like most TNF-related ligands however, TRAIL has been demonstrated to exert pleiotropic functions including abilities to trigger cell proliferation or differentiation. ${ }^{13,14}$ Apoptosis triggering by TRAIL involves principally two receptors, DR4 and DR5 also known as TRAIL-R1 and TRAIL$\mathrm{R} 2$, which upon binding to their cognate ligand engage the recruitment of the adaptor protein FADD and initiator caspases (caspase-8 and/or -10) via homotypic interactions through the death-domain and the death-effector-domains, respectively, leading to the formation of the socalled DISC (death inducing signaling complex). ${ }^{15}$ Notably, the TRAIL ligand/ receptor system differs between mouse and humans. ${ }^{16,17}$ For example, only one TRAIL receptor containing a death-domain has been identified in the mouse.
Correspondence: Michael Hahne, Institut de Génétique Moléculaire de Montpellier, CNRS, UMR5535, 1919 Route de Mende, 34293 Montpellier Cedex 5, France.

E-mail: hahne@igmm.cnrs.fr

Key words: TRAIL, rheumatoid arthritis.

Acknowledgements: This study and JM and MH were supported by PROA (INSERM) and ARC (Association pour la Recherche sur le Cancer). RA is supported by the société Française de Rhumatologie (SFR) and MH by the contrat interface no. 05.524-DRV/MC/SS. NL is supported by a doctoral joint fellowship from INSERM: Conseil Régional de Bourgogne and ARC. OM is supported by research grants from ARC, ANR (Agence Nationale de la Recherche, ANR-06-JCJC-0103 et ANR-07-PCVI-0031), and the European Community (ApopTrain Marie Curie RTN).

Received for publication: 8 June 2009 .

Revision received: 14 October 2009.

Accepted for publication: 14 October 2009.

This work is licensed under a Creative Commons Attribution 3.0 License (by-nc 3.0).

(C) Copyright N. Lalaoui et al., 2009

Licensee PAGEPress, Italy

Rheumatology Reports 2009; 1:e10

doi:10.4081/rr.2009.e10

In type I cells, DISC formation and caspase8 activation generally is sufficient to promote caspase- 3 processing and apoptosis triggering. In type II cells, DISC formation and caspase-8 activation are weaker compared to type I cells, and full caspase- 3 activation occurs through the mitochondrial amplification loop via Bid cleavage. ${ }^{18}$ Efficient DISC engagement is the limiting factor that defines caspase- 8 activation and thus cellular dependency to type I or II signaling pathways. Accordingly, it has been demonstrated that the mitochondrial requirement in some type II cells could be overcome by increasing the concentrations of TRAIL. ${ }^{19}$ Likewise, negative regulation of TRAIL DISCinduced formation occurs in cells that express DcR1 or DcR2, also coined TRAIL-R3 and TRAIL-R4. ${ }^{20}$ DcR1 and DcR2 are devoid of a functional death-domain and impair TRAILinduced cell death either directly by competing with their cognate ligand, or through regulatory inhibitory activity leading to reduced caspase-8 processing within the TRAIL DISC. ${ }^{20,21}$ Similar to DcR1, the soluble receptor OPG, albeit exhibiting the lowest affinity for TRAIL among the TRAIL receptors, is able to compete for TRAIL binding and thus impair TRAILinduced signaling. ${ }^{22,23}$

The TRAIL system is probably one of the most complex members of the TNF family, owing to the large number of receptors to which TRAIL can bind and to the signaling 
pathways that are engaged. DR4 and DR5 are both capable of triggering apoptosis and are the receptors that exhibit the highest affinity for TRAIL. ${ }^{24}$ The existence of two apparently redundant receptors may have physiological meaning and it is thought that they could play distinct roles with respect to the control of tissue homeostasis or activation of secondary signaling pathways. Accordingly, while most cancer cell lines engage either DR4 or DR5 to trigger TRAIL-induced apoptosis, ${ }^{25}$ chronic Bcell leukemia cells engage DR4 preferentially despite co-expressing DR5, ${ }^{26}$ and some colon carcinoma cell lines preferentially engage DR5. ${ }^{27}$ The reasons for these specific engagements are not clear. Recently DR4- and DR5mediated apoptosis were shown to be controlled by 0 -glycosylation, ${ }^{28}$ a finding that could help explain the specific engagement or the importance of each agonistic receptor with respect to TRAIL-induced signaling pathways. Indeed, some DR5 "specific" TRAIL mutants revealed that the JNK pathway is preferentially activated by DR5. ${ }^{29}$

Besides the TRAIL membrane-bound complex I or DISC, TRAIL was recently demonstrated to initiate the formation of a secondary complex, which contrarily to $\mathrm{TNF}^{30}$ is thought to be required to trigger nonapoptotic signaling activities including the activation of the NF-kB, JNK, or p38 pathways. ${ }^{31}$ TRAIL has been described to induce cell proliferation or cell survival in many cell types including vascular smooth muscle cells, synovial fibroblasts, vascular endothelial cells, or cancer cells, ${ }^{32-38}$ as well as to induce intestinal, skeletal myoblast, or osteoclast differentiation. ${ }^{13,14,39}$ Animal models in which TRAIL expression has been inactivated demonstrated that TRAIL plays a crucial role in immune tumor surveillance ${ }^{40}$ and that it could contribute to the control of some autoimmune diseases ${ }^{41,42}$ including rheumatoid arthritis.

\section{TRAIL in rheumatoid arthritis}

The first report linking TRAIL with arthritis came from a mouse study using the collageninduced arthritis (CIA) model..$^{41,43}$ In this model susceptible mouse strains with type II collagen (CII) from heterologous species lead to a pathology that resembles human RA called collagen-induced arthritis (CIA) ${ }^{44}$ including footpad swelling, synovitis, pannus formation, and bone and cartilage destruction of paw joints. Both CII antibodies and CD4 ${ }^{+}$T-cells are required for the development of CIA, and the classical mouse strain used for the CIA model is DBA/1 mice. Using the CIA model Song and co-workers observed that blockade of TRAIL in mice using a soluble recombinant receptor exacerbated autoimmune arthritis, whereas

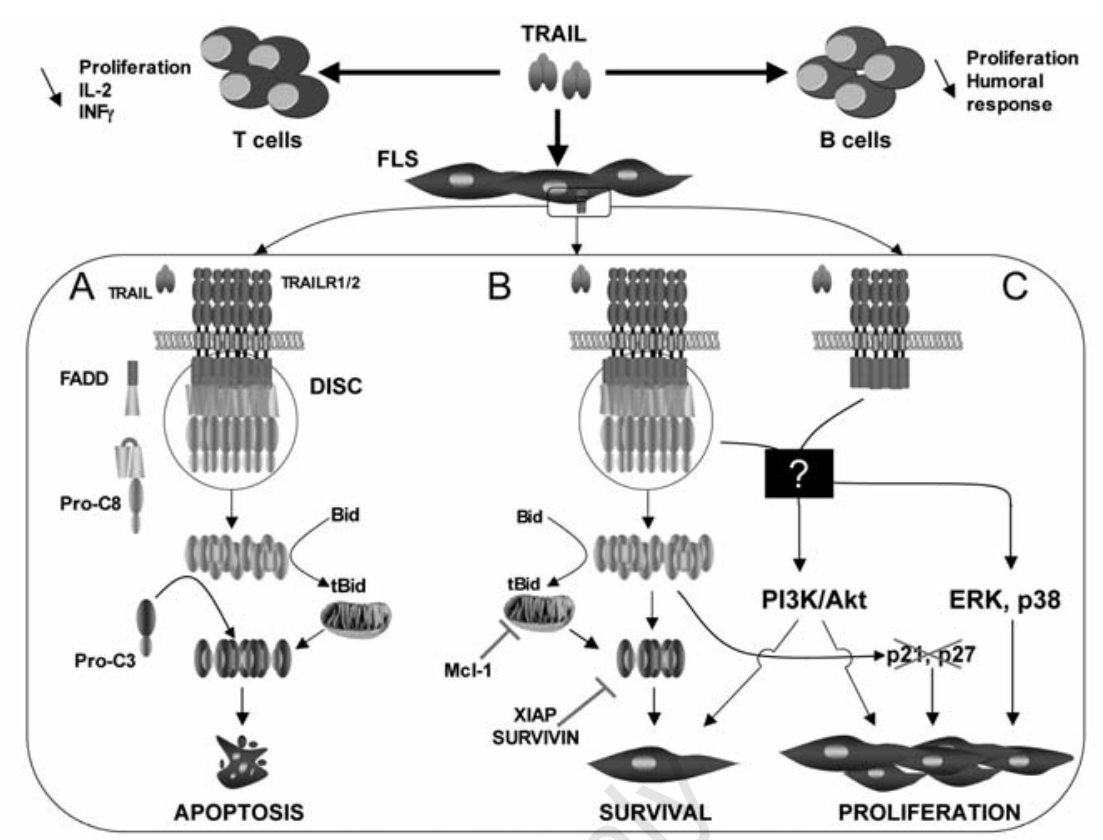

Figure 1. The complex role of TRAIL in rheumatoid arthritis. TRAIL appears to control T- and B-cell responses. On human synovial fibroblasts (FLS), TRAIL acts as a pleiotropic cytokine: (A) TRAIL can trigger apoptosis by forming the DISC in FLS cells. Activated caspase-8 initiates apoptosis by direct cleavage of downstream effector caspase-3 (ProC3) and Bid. (B) FLS overexpressing antiapoptotic molecules such as Mcl-1 or IAPs members, XIAP or survivin for example, are resistant toward TRAIL-induced apoptosis and survive. Activation of the PI3 kinase/Akt signaling pathway mediates FLS survival as well. (C) FLS can proliferate upon TRAIL treatment by activating PI3K and MAPK pathways. Activation of caspase-8 triggers FLS proliferation by cleavage of the cell cycle inhibitors p21 and p27.

intra-articular TRAIL adenoviral gene transfer diminished the symptoms. ${ }^{43}$ Blockage of TRAIL resulted in an increased proliferation of synoviocytes and intra-articular lymphocytes. Moreover, the authors could demonstrate that TRAIL prevented cell cycle progression of lymphocytes in vitro. The capacity of TRAIL to control CIA was confirmed using TRAIL $^{-1}$ mice. ${ }^{41}$ TRAIL $^{-1}$ C57BL/6 mice developed severe arthritis indistinguishable from arthritis in DBA1, whereas TRAIL $^{+++}$C57BL/6 mice were not susceptible to CIA owing to their genetic background. Mice deficient for TRAIL were found to have increased cellular and humoral immune responses against self-antigens. Two other studies employed adenoviral-mediated gene transfer of TRAIL in animal models of arthritis. In an Il-1 $\beta$ triggered rabbit model of arthritis intra-articular adenoviral TRAIL delivery was shown to modulate inflammation. ${ }^{45}$ In this study the attenuation of inflammation was correlated with TRAIL-induced apoptosis in cells within the synovium. The same authors obtained similar results by using recombinant TRAIL instead of TRAIL-encoding adenovirus. ${ }^{46}$ In a preclinical study by Liu et al. dendritic cells were transduced with an adenovirusbased vector able to express TRAIL and pulsed with collagen, the autoantigen responsible for disease in the CIA model. The primed dendritic cells were predicted to specifically interact and thus eliminate, only those T-lymphocytes that recognize the collagen. Indeed, treatment with TRAIL-expressing collagen-pulsed dendritic cells limited the incidence of arthritis in the CIA model by modulating T-cell responses and correlated with detection of apoptotic Tcells in the spleen. In addition, B-cell responses were affected as titers of anti-collagen II antibodies were lower. Taken together, the results obtained in animal models for arthritis support the concept that TRAIL has a therapeutic potential although the underlying mechanisms remain to be clarified.

The pseudo-tumoral proliferation of RA FLS is considered to be the major mechanism for the hyperplasic growth of the RA synovium and can be mimicked by in vitro culturing, since $e x$ vivo RA FLS cells grow in normal medium without requiring additional stimulation. Various groups have tested how TRAIL does modulate these RA FLS cultures: a study by 
Ichikawa et al. described TRAIL-R1 and -R2 expression on primary isolated FLS from RA patients and analyzed the effect of TRAIL on RA FLS for short culturing periods; that is, up to 24 hours. Varying levels of apoptosis were induced by TRAIL on the different RA FLS cultures tested, in which a portion of cells survived. ${ }^{47}$ These RA FLS strongly expressed TRAIL-R2 and were highly susceptible to an agonistic anti-TRAIL-R2 antibody, identifying TRAIL-R2 as the receptor mediating TRAILinduced apoptosis. ${ }^{47}$ These observations are in agreement with a report by Miranda-Carus et $a l .{ }^{48}$ In this study, 50 fibroblasts from RA synovial fluid samples were analyzed and about half of them were found to express TRAIL-R2. These cells underwent apoptosis when treated in vitro with an agonistic anti-TRAIL-R2 antibody. ${ }^{48}$ While these reports suggested the specific targeting of TRAIL-R2 on RA FLS as a potential therapeutic approach, Perlman et al. drew the opposite conclusion because they could not detect the expression of TRAIL-R1 or TRAIL-R2, or the susceptibility to TRAIL in RA FLS. ${ }^{49}$ Another study by Park et al. concluded that cultured FLS are not sensitive to TRAILinduced apoptosis in spite of TRAIL-R1 expression, whereas FLS became sensitive in the presence of actinomycin D or cycloheximide..$^{50}$ One report correlated resistance of RA FLS for TRAIL-induced apoptosis with expression levels of TRAIL-R4, as TRAIL-R4 siRNA could sensitize FLS. ${ }^{51}$

Our group has analyzed in two studies TRAIL-responses of RA FLS. ${ }^{35,52}$ We detected expression of the TRAIL receptors 1 and 2 on FLS, and described that both recombinant TRAIL as well as an agonistic anti-TRAIL-R2 antibody induces apoptosis only in a subset of RA FLS that is followed by an induction of proliferation in the surviving cells..$^{35}$ Notably, the observed dual functionality of TRAIL on RA FLS concurs with the previously reported pleiotropic responses of TRAIL in primary human tumor cells..$^{35,53}$ We observed a variation in TRAIL-sensitivity of RA FLS according to the patients from which they are derived, which is in agreement with the report of Ichikawa et al. ${ }^{47}$

A possible explanation for the opposing observations on TRAIL-sensitivity of RA FLS might be the use of different protocols to isolate and/or culture synovial fibroblasts obtained from biopsies. For example, RA FLS were shown to produce the endogenous decoy receptor OPG that can interfere with the efficiency of recombinant TRAIL on cell cultures that have not received fresh medium. ${ }^{22}$ Another important factor appears to be the cell cycle dependency of RA FLS for TRAIL-induced apoptosis. ${ }^{54}$ The discrepancy in reported TRAIL receptor expression might be because of the different antibodies used.

While most studies focus on TRAIL responses on synovial fibroblasts, one study analyzed synovial T-cells. ${ }^{55}$ Lorenzo et al. reported that synovial fluid T-cells from RA patients are sensitive to TRAIL- but not Fas ligand-induced apoptosis. Several reports characterized TRAIL-induced signaling in RA FLS. The group of Zhang analyzed FLS treated with the proteasome inhibitor lactacystin and described a specific role for p53 in TRAIL-R2-mediated apoptosis. Under these conditions p53 siRNA was able to reduce TRAIL-induced apoptosis. ${ }^{56}$ Caspase- 4 was found to induce cleavage of vimentin associated in a complex with p53, thus releasing $\mathrm{p} 53$. We could demonstrate the involvement of the ERK p38 and PI3 kinase/Akt signaling pathways in TRAIL-induced RA FLS proliferation, but that only PI3 kinase/Akt protects RA FLS from TRAIL-triggered apoptosis. In line with this is a study by Miyashita et al. reporting that Akt renders RA FLS resistant to TRAIL-induced apoptosis. ${ }^{57}$ Moreover, we found that not only TRAIL-induced apoptosis, but also TRAIL-triggered proliferation in RA FLS is mediated by caspases via degradation of the cell cycle inhibitors p21 and p27. We therefore suggest that caspases act synergistically with PI3 kinase and/or MAP kinases to mediate TRAIL-induced proliferation in RA FLS. A correlation of p21 expression levels with cell viability in TRAIL-treated RA FLS has been concluded also by Juengel et al. These authors showed that the histone deacetylases inhibitor trichostatin sensitizes RA FLS for TRAILinduced cell death and induced cell cycle arrest by upregulating p 21 levels. ${ }^{58}$

In summary, a pattern is emerging indicating that TRAIL acts as a pleiotropic cytokine on tumor-like human RA FLS by inducing different responses (Figure 1). This differs from previous reports describing that TRAIL has a protective role in the collagen-induced mouse model of arthritis by blocking the proliferation of synovial cells. This discrepancy could reflect the different pathogenic mechanisms between RA in the joints of patients and the respective mouse model. Moreover, the organization of TRAIL signaling between human and mouse appears to be different, as only one membrane-anchored TRAIL receptor and two soluble decoy receptors have been identified in the mouse. One recent report compared TRAIL and TRAIL receptor expression in synovial tissues of RA, osteoarthritis, and spondyloarthritis patients by immunohistochemistry and found the highest expression for TRAIL and its membrane-bound receptors in tissues of RA patients. ${ }^{59}$ Moreover, increased levels of the apoptosis inhibitors survivin and xIAP were found in synovial tissues of patients with active RA when compared with those of inactive RA. This finding could explain not only the opposing effects of TRAIL on cultured RA FLS but also suggests that resistance for TRAILinduced apoptosis correlates with disease severity.

\section{References}

1. Firestein GS. Evolving concepts of rheumatoid arthritis. Nature 2003;423: 356-61.

2. Pope RM. Apoptosis as a therapeutic tool in rheumatoid arthritis. Nat Rev Immunol 2002;2:527-35.

3. Aggarwal BB. Signalling pathways of the tnf superfamily: A double-edged sword. Nat Rev Immunol 2003;3:745-56.

4. Wang S, El-Deiry WS. Trail and apoptosis induction by tnf-family death receptors. Oncogene 2003;22:8628-33.

5. Ashkenazi A, Pai RC, Fong S, et al. Safety and antitumor activity of recombinant soluble apo2 ligand. J Clin Invest 1999;104: 155-62.

6. Herbst RS, Mendolson DS, Ebbinghaus S, et al. A phase 1 safety and pharmacokinetic (pk) study of recombinant apo2l/trail, an apoptosis-inducing protein in patients with advanced cancer. J Clin Oncol 2006; 24:3013.

7. Plummer R, Attard G, Pacey S, et al. Phase 1 and pharmacokinetic study of lexatumumab in patients with advanced cancers. Clin Cancer Res 2007;13:6187-94.

8. Hotte SJ, Hirte HW, Chen EX, et al. A phase 1 study of mapatumumab (fully human monoclonal antibody to trail-r1) in patients with advanced solid malignancies. Clin Cancer Res 2008;14:3450-5.

9. Boorjian SA, Milowsky MI, Kaplan J, et al. Phase 1/2 clinical trial of interferon alpha2b and weekly liposome-encapsulated all-trans retinoic acid in patients with advanced renal cell carcinoma. J Immunother 2007;30:655-62.

10. Greco FA, Bonomi P, Crawford J, et al. Phase 2 study of mapatumumab, a fully human agonistic monoclonal antibody which targets and activates the trail receptor-1, in patients with advanced non-small cell lung cancer. Lung Cancer 2008;61:8290.

11. Tolcher AW, Mita M, Meropol NJ, et al. Phase 1 pharmacokinetic and biologic correlative study of mapatumumab, a fully human monoclonal antibody with agonist activity to tumor necrosis factor-related apoptosis-inducing ligand receptor-1. J Clin Oncol 2007;25:1390-5.

12. Wilson NS, Dixit V, Ashkenazi A. Death receptor signal transducers: Nodes of coordination in immune signaling networks. Nat Immunol 2009;10:348-55.

13. Yen ML, Tsai HF, Wu YY, et al. Tnf-related apoptosis-inducing ligand (trail) induces osteoclast differentiation from monocyte/macrophage lineage precursor cells. Mol Immunol 2008;45:2205-13.

14. Rimondi E, Secchiero P, Quaroni A, et al. 
Involvement of trail/trail-receptors in human intestinal cell differentiation. J Cell Physiol 2006;206:647-54.

15. Merino D, Lalaoui N, Morizot A, et al. Trail in cancer therapy: present and future challenges. Expert Opin Ther Targets 2007;11: 1299-314.

16. Schneider P, Olson D, Tardivel A, et al. Identification of a new murine tumor necrosis factor receptor locus that contains two novel murine receptors for tumor necrosis factor-related apoptosis-inducing ligand (trail). J Biol Chem 2003;278:544454.

17. Yagita H, Takeda K, Hayakawa Y, et al. Trail and its receptors as targets for cancer therapy. Cancer Sci 2004;95:777-83.

18. Ozoren N, El-Deiry WS. Defining characteristics of types I and II apoptotic cells in response to trail. Neoplasia NY 2002;4:5517.

19. Rudner J, Jendrossek V, Lauber K, et al. Type I and type II reactions in trail-induced apoptosis: results from dose-response studies. Oncogene 2005;24:130-40.

20. Merino D, Lalaoui N, Morizot A, et al. Differential inhibition of trail-mediated dr5-disc formation by decoy receptors 1 and 2. Mol Cell Biol 2006;26:7046-55.

21. Clancy L, Mruk K, Archer K, et al. Preligand assembly domain-mediated ligand-independent association between trail receptor $4(\operatorname{tr} 4)$ and tr2 regulates trail-induced apoptosis. Proc Natl Acad Sci USA, 2005.

22. Miyashita T, Kawakami A, Nakashima T, et al. Osteoprotegerin (opg) acts as an endogenous decoy receptor in tumour necrosis factor-related apoptosis-inducing ligand (trail)-mediated apoptosis of fibroblast-like synovial cells. Clin Exp Immunol 2004;137:430-6.

23. Zerafa N, Westwood JA, Cretney E, et al. Cutting edge: Trail deficiency accelerates hematological malignancies. J Immunol 2005;175:5586-90.

24. Truneh A, Sharma S, Silverman C, et al. Temperature-sensitive differential affinity of trail for its receptors. Dr5 is the highest affinity receptor. J Biol Chem 2000;275: 23319-25.

25. Bin L, Thorburn J, Thomas LR, et al. Tumor-derived mutations in the trail receptor dr5 inhibit trail signaling through the $\mathrm{dr} 4$ receptor by competing for ligand binding. J Biol Chem 2007;282:28189-94.

26. MacFarlane M, Harper N, Snowden RT, et al. Mechanisms of resistance to trailinduced apoptosis in primary B cell chronic lymphocytic leukaemia. Oncogene 2002; 21:6809-18.

27. Wang S, El-Deiry WS. Inducible silencing of killer/dr5 in vivo promotes bioluminescent colon tumor xenograft growth and confers resistance to chemotherapeutic agent 5-fluorouracil. Cancer Res 2004;64: 6666-72.

28. Wagner KW, Punnoose EA, Januario T, et al. Death-receptor 0-glycosylation controls tumor-cell sensitivity to the proapoptotic ligand apo2//trail. Nat Med 2007;13:1070-7.

29. Muhlenbeck F, Schneider P, Bodmer JL, et al. The tumor necrosis factor-related apoptosis-inducing ligand receptors trail-r1 and trail-r2 have distinct cross-linking requirements for initiation of apoptosis and are non-redundant in jnk activation. $\mathrm{J}$ Biol Chem 2000;275:32208-13.

30. Micheau 0, Tschopp J. Induction of tnf receptor 1-mediated apoptosis via two sequential signaling complexes. Cell 2003;114:181-90.

31. Varfolomeev E, Maecker H, Sharp D, et al. Molecular determinants of kinase pathway activation by apo 2 ligand/tumor necrosis factor related apoptosis-inducing ligand. J Biol Chem 2005;280:40599-608.

32. Belyanskaya LL, Ziogas A, HopkinsDonaldson S, et al. Trail-induced survival and proliferation of sclc cells is mediated by erk and dependent on trail-r2/dr5 expression in the absence of caspase- 8 . Lung Cancer 2008;60:355-65.

33. Ehrhardt H, Fulda S, Schmid I, et al. Trail induced survival and proliferation in cancer cells resistant towards trail-induced apoptosis mediated by nf-kappab. Oncogene 2003;22:3842-52.

34. Kavurma MM, Schoppet M, Bobryshev YV, et al. Trail stimulates proliferation of vascular smooth muscle cells via activation of nf-kappab and induction of insulin-like growth factor-1 receptor. J Biol Chem 2008;283:7754-62.

35. Morel J, Audo R, Hahne M, et al. Tumor necrosis factor-related apoptosis-inducing ligand (trail) induces rheumatoid arthritis synovial fibroblast proliferation through mitogen-activated protein kinases and phosphatidylinositol 3-kinase/akt. J Biol Chem 2005;280:15709-18.

36. Secchiero P, Gonelli A, Carnevale E, et al. Trail promotes the survival and proliferation of primary human vascular endothelial cells by activating the akt and erk pathways. Circulation 2003;107:2250-6.

37. Secchiero P, Zerbinati C, Rimondi E, et al. Trail promotes the survival, migration and proliferation of vascular smooth muscle cells. Cell Mol Life Sci 2004;61:1965-74.

38. Vilimanovich U, Bumbasirevic V. Trail induces proliferation of human glioma cells by c-flipl-mediated activation of erk1/2. Cell Mol Life Sci 2008;65:814-26.

39. O'Flaherty J, Mei Y, Freer M, et al. Signaling through the trail receptor dr5/fadd pathway plays a role in the apoptosis associated with skeletal myoblast differentiation. Apoptosis 2006;11:2103-13.
40. Takeda K, Smyth MJ, Cretney E, et al. Critical role for tumor necrosis factorrelated apoptosis-inducing ligand in immune surveillance against tumor development. J Exp Med 2002;195:161-9.

41. Lamhamedi-Cherradi SE, Zheng SJ, Maguschak KA, et al. Defective thymocyte apoptosis and accelerated autoimmune diseases in trail(-/-) mice. Nat Immunol 2003;4:255-60.

42. Anel A, Bosque A, Naval J, et al. Apo2l/trail and immune regulation. Front Biosci 2007; 12:2074-84.

43. Song K, Chen Y, Goke R, et al. Tumor necrosis factor-related apoptosis-inducing ligand (trail) is an inhibitor of autoimmune inflammation and cell cycle progression. J Exp Med 2000;191:1095-104.

44. Courtenay JS, Dallman MJ, Dayan AD, et al. Immunisation against heterologous type II collagen induces arthritis in mice. Nature 1980;283:666-8.

45. Yao Q, Wang $S$, Gambotto A, et al. Intraarticular adenoviral-mediated gene transfer of trail induces apoptosis of arthritic rabbit synovium. Gene Ther 2003;10:105560.

46. Yao Q, Seol DW, Mi Z, et al. Intra-articular injection of recombinant trail induces synovial apoptosis and reduces inflammation in a rabbit knee model of arthritis. Arthritis Res Ther 2006;8:R16.

47. Ichikawa K, Liu W, Fleck M, et al. Trail-r2 (dr5) mediates apoptosis of synovial fibroblasts in rheumatoid arthritis. J Immunol 2003;171:1061-9.

48. Miranda-Carus ME, Balsa A, BenitoMiguel M, et al. Rheumatoid arthritis synovial fluid fibroblasts express trail-r2 (dr5) that is functionally active. Arthritis Rheum 2004;50:2786-93.

49. Perlman H, Nguyen N, Liu H, et al. Rheumatoid arthritis synovial fluid macrophages express decreased tumor necrosis factor-related apoptosis-inducing ligand r2 and increased decoy receptor tumor necrosis factor-related apoptosisinducing ligand $r 3$. Arthritis Rheum 2003;48:3096-101.

50. Park YW, Ji JD, Lee JS, et al. Actinomycin $\mathrm{d}$ renders cultured synovial fibroblasts susceptible to tumour necrosis factor related apoptosis-inducing ligand (trail)-induced apoptosis. Scand J Rheumatol 2003;32: 356-63.

51. Terzioglu E, Bisgin A, Sanlioglu AD, et al. Concurrent gene therapy strategies effectively destroy synoviocytes of patients with rheumatoid arthritis. Rheumatology 2007; 46:783-9.

52. Audo R, Combe B, Coulet B, et al. The pleiotropic effect of trail on tumor-like synovial fibroblasts from rheumatoid arthritis patients is mediated by caspases. Cell 
Death Differ 2009;16:1227-37.

53. Baader E, Toloczko A, Fuchs U, et al. Tumor necrosis factor-related apoptosis-inducing ligand-mediated proliferation of tumor cells with receptor-proximal apoptosis defects. Cancer Res 2005;65:7888-95.

54. Pundt N, Peters MA, Wunrau C, et al. Susceptibility of rheumatoid arthritis synovial fibroblasts to fasl- and trail-induced apoptosis is cell cycle-dependent. Arthritis Res Ther 2009;11:R16.

55. Martinez-Lorenzo MJ, Anel A, Saez-
Gutierrez B, et al. Rheumatoid synovial fluid $\mathrm{T}$ cells are sensitive to apo2l/trail. Clin Immunol 2007;122:28-40.

56. Zhang HG, Wang J, Yang X, et al. Regulation of apoptosis proteins in cancer cells by ubiquitin. Oncogene 2004;23:200915.

57. Miyashita T, Kawakami A, Tamai M, et al. Akt is an endogenous inhibitor toward tumor necrosis factor-related apoptosis inducing ligand-mediated apoptosis in rheumatoid synovial cells. Biochem
Biophys Res Commun 2003;312:397-404.

58. Jungel A, Baresova V, Ospelt C, et al. Trichostatin a sensitises rheumatoid arthritis synovial fibroblasts for trailinduced apoptosis. Ann Rheum Dis 2006; 65:910-2.

59. Dharmapatni AA, Smith MD, Findlay DM, et al. Elevated expression of caspase-3 inhibitors, survivin and xiap correlates with low levels of apoptosis in active rheumatoid synovium. Arthritis Res Ther 2009;11:R13. 